\title{
Bolivia has the opportunity to create the planet's richest park for terrestrial biota
}

\author{
J. V. REMSEN, JR, and T. A. PARKER, III
}

\begin{abstract}
Summary
Establishing a reserve of approximately $10,000 \mathrm{~km}^{2}$ at a strategic location in depto. La Paz, northern Bolivia, would create the planet's richest park for birds and presumably all other forest-dwelling biota that reach peak diversity at tropical latitudes. By connecting puna and montane forest habitats of the Andes with lowland tropical forest and savannas of the Amazon basin, we predict that the proposed reserve would contain at least 1,088 bird species, or roughly $11 \%$ of all bird species on the planet. Among these are many threatened species and species with relatively small geographical ranges. The proposed reserve would also include threatened habitats, such as lower montane forest, dry forest, and grassland.
\end{abstract}

El establecimiento de una reserva de aproximadamente $10.000 \mathrm{~km}^{2}$ en un lugar estratégico en el departamento de La Paz, Bolivia, crearía el parque de major riqueza de aves en el planeta. Así es de suponer que la flora y fauna que habitan en la área, llegan a su grado máximo de diversidad en las latitudes tropicales. Conectando puna, los hábitats de bosque tropical (áreas bajas) y sabanas de la cuenca del Amazonas, predecimos que la reserva que se propone tendría a lo menos 1.088 especies de aves, 0 aproximadamente $11 \%$ de todas las especies de aves del planeta. Dentro de estas hay muchas especies amenazadas y especies con áreas de rango geográfico muy restringido. Además la reserva que se propone también incluiría hábitats amenazados, como bosque montano, bosque seco y pajonales tropicales.

\section{Introduction}

It is well known that the lowland forests of western Amazonia and the humid montane forests of the eastern slope of the Andes are among the richest habitats in the world for species of terrestrial organisms. Thus, any park that bridged these adjacent habitats and was placed anywhere from central Colombia to northern Bolivia would be one of the planet's richest parks. If such a park could be extended to include both páramo and puna zones of the Andes, which is possible in Peru and northern Bolivia, then it would be the world's richest park. If such a park could also include some of the open lowland or drier habitats characteristic of southern South America, then this park would include not only more species of organisms than any other park on the planet, but also more species than exist in almost any country in the world as well, all within a relatively small area. In addition to astonishing species richness, the hypothetical park would encompass threatened habitats and many threatened species. 


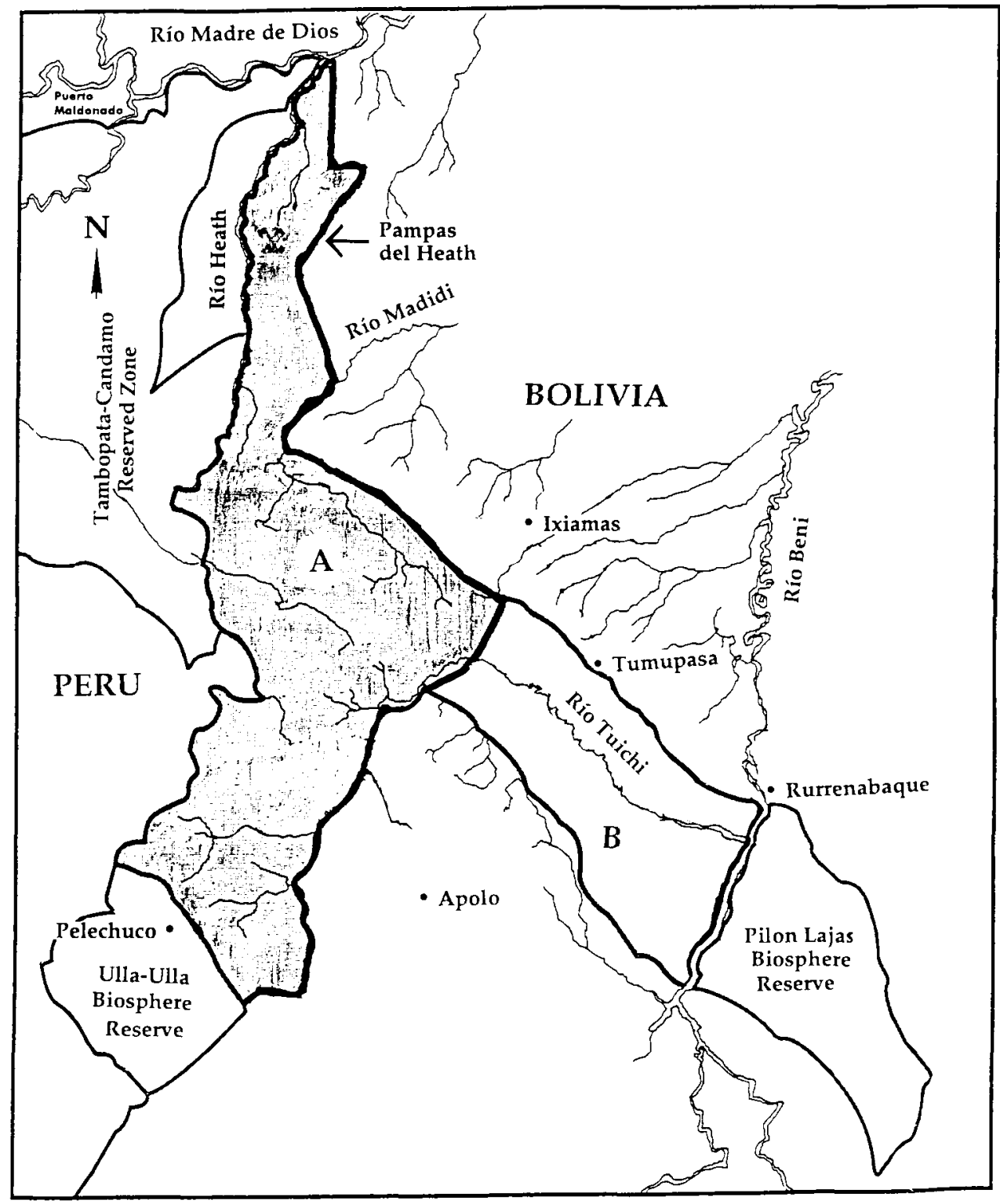

Figure 1. Location of proposed park or reserve system (shaded area, A) in depto. La Paz, northern Bolivia. Addition of the majority the Río Tuichi drainage (B) would connect the proposed park to the Pilon Lajas Biosphere Reserve. (Figure modified from one by S. F. Moolenijzer for TREX.)

The purpose of this paper is to present calculations of potential species richness for one group of terrestrial organisms, birds, for an area in extreme northern Bolivia near the border with Peru in depto. La Paz. The boundaries for the hypothetical park extend from an existing reserve, the Ulla-Ulla Biosphere Reserve, over the Andes to the area for the proposed Alto Madidi reserve and then north to the Pampas del Heath (Figure 1). The size of such a park is 
approximately $10,000 \mathrm{~km}^{2}$. This area would include relatively undisturbed puna and altiplano habitats in Ulla-Ulla, the páramo zone of the humid slope of the Andes, humid forest of the Andes from timberline to the lowlands, forested outlying ridges of the Andes, humid lowland forest, successional vegetation along major rivers, montane deciduous forest in the central Tuichi and Machariapo valleys, and one of the largest, least disturbed, ungrazed grasslands in the world, the Bolivian Pampas del Heath (Parker and Bailey 1991). Inclusion of this range of habitat diversity and this degree of pristine quality of the habitats in the region is probably not possible elsewhere on the planet within such a relatively small area (slightly larger than Yellowstone National Park, U.S.A., but smaller than Northern Ireland, Gambia, Jamaica, Hawaii or Connecticut). A park that spans a continuous elevational gradient from lowlands to snow-line also permits seasonal elevational movements of birds and other mobile organisms.

\section{Methods}

Our calculations are based on the bird species already recorded in the region and those that we predict will be found there once additional habitats and localities are sampled. Species predicted to occur in the park are assigned one of two levels of uncertainty: "expected" for those species recorded both north and south of the park but not yet within it, or recorded only on one side of the park but as close as $100 \mathrm{~km}$ in habitats present in the proposed park; and "possible" for those species recorded from 100 to $200 \mathrm{~km}$ beyond the proposed park boundaries but in habitats found within the park.

Sources for species already recorded within the proposed boundaries are: Ribera and Hanagarth (1982) for the Ulla-Ulla Biosphere Reserve; Parker and Bailey (1991) for the Alto Madidi site; T.A.P.'s data from the Bolivian side of the Río Heath as presented in Remsen and Traylor (1989) and Parker et al. (1991); and Whitney (1994) for a locality at c.3,000 m near Pelechuco, depto. La Paz, Bolivia. Sources for species "expected" to occur are as follows: (1) Graham et al. (1980), Foster et al. (1994), and unpublished data from the Museum of Natural Science, Louisiana State University (LSUMZ), and Museo Javier Prado, Lima, for the Peruvian side of the Pampas del Heath, depto. Madre de Dios, Peru; (2) Parker and Bailey (1991) for the savanna at Ixiamas and lower montane forest at Calabatea, depto. La Paz, Bolivia; (3) S. W. Cardiff et al. (unpubl. data) for lower montane forest in the Cerro Asunto Pata area, depto. La Paz, Bolivia, where a locality at 1,300 $\mathrm{m}$ was sampled in July and August 1993 by a team from the LSUMZ and the Museo Nacional de Historia Natural, La Paz; (4) unpublished and some published (Remsen 1984) specimen data from humid montane forest at Abra de Maruncunca, 2,000 m, and Valcón, 3,000 m, depto. Puno, from the LSUMZ and Museo Javier Prado, Lima, these localities lying within about $35 \mathrm{~km}$ of the Bolivian border; and (5) general distributional data from depto. La Paz from Remsen and Traylor (1989). Sources for species of "possible" occurrence in the area are Remsen and Traylor (1989) and Fjeldså and Krabbe (1990) for depto. La Paz and depto. Puno, and Foster et al. (1994) for lowlands of depto. Madre de Dios and isolated ridges of depto. Puno, south-east Peru. 
To assess the number of bird species with "small" geographical ranges that might be contained within the proposed reserve, we consulted references on South American bird distribution (e.g. Fjeldså and Krabbe 1990). We arbitrarily considered any species of Andean bird whose distribution includes only extreme southern Peru (depto. Puno) and northern Bolivia (deptos. La Paz, Cochabamba, and extreme northern Santa Cruz) to have a "small" range. For lowland species, we considered distribution that included only south-east Peru (deptos. Madre de Dios or Puno) and northern Bolivia (deptos. Pando, La Paz, and northern Beni) as "small". To determine the number of endangered, threatened, and potentially threatened species, we used appendix B of Collar et al. (1992). We condensed their 12 categories into five categories by using their alternative descriptive terms: (1) Endangered, (2) Indeterminate, (3) Vulnerable (including "vulnerable/rare"), (4) Rare (no species from Appendix in this category), and (5) Insufficiently Known.

\section{Results and discussion}

Our calculations (Appendix) show that a reserve system delineated as in Figure I would contain a minimum of 1,088 bird species (recorded or expected), or roughly $11 \%$ of all bird species on the planet. Inclusion of the "possible" category would boost the total to 1,138 species. Even this total may be conservative, because range extensions of more than $200 \mathrm{~km}$ are a routine feature of avifaunal surveys in South America (e.g. Parker and Remsen 1987). Nonetheless, this total is more than the number of bird species recorded in all but a few countries in the world. We predict that similar calculations for other groups of terrestrial biota that reach peak species richness in the Neotropical region, particularly in forest habitats, would show the same pattern: such a park would be the richest in the world for them as well.

Species richness is only one measure of the biotic value of a reserve. Another is the number of species with small geographical ranges. Using our criteria for "small" geographical ranges (see Methods), the proposed park has seven such species recorded, 17 "expected" to be recorded, and three "possible". Another criterion is the number of endangered or threatened species. Using Collar et al. (1992; see Methods), eight such species are "expected" to be recorded and six are "possible" in the proposed park (Table 1). This total of 17 species is more

Table 1 . Threatened bird species of the proposed park in northern Bolivia. See Methods for explanations for categories for conservation status and occurrence.

\begin{tabular}{lll}
\hline Conservation status & \multicolumn{1}{c}{ Expected } & Occurrence \\
\cline { 2 - 3 } Endangered & Cinclodes aricomae & Possible \\
Indeterminate & Asthenes berlepschi & $\begin{array}{l}\text { Simoxenops striatus } \\
\text { Anairetes alpinus }\end{array}$ \\
Vulnerable & $\begin{array}{l}\text { Crax globulosa } \\
\text { Popelairia letitiae }\end{array}$ \\
& Nothoprocta taczanowskii & Pauxi unicomis \\
& Terenura sharpei & \\
& Myrmotherula grisea \\
& Formicarius rufifrons \\
Agriornis andicola & \\
& Tinamus osgoodi & \\
\hline
\end{tabular}


than the national totals for 25 of the 34 countries of the Western Hemisphere (using totals from appendix $C$ in Collar et al. 1992). The proposed park would also contain the entire known range of the newly discovered, undescribed Herpsilochmus antwren (Pearman 1993).

More broadly, the proposed park contains at least two endangered habitats. Inclusion of the grasslands of the Pampas del Heath would protect one of the last ungrazed grasslands in South America (Parker and Bailey 1991) and perhaps the most pristine major area of grassland on the continent. Inclusion of lower montane cloud-forest would protect a habitat that is everywhere threatened in the Neotropics (Parker and Bailey 1991). If the proposed park contains Polylepis woodland, then it would also protect another endangered habitat (Fjeldså 1992).

We encourage the Bolivian government and non-governmental conservation and development agencies to work together to make the proposed park a reality and a source of national pride. Although access to most of the proposed park is currently difficult, visits to parts of the park for ecotourism will become more feasible when the La Paz to Apolo road is completed. Access from Rurrenabaque through the Río Tuichi is also a possibility. The lure provided by advertising that the park is the richest in the world should accelerate development of access for ecotourism and provide economic incentive for establishing the park.

\section{Acknowlegements}

The National Geographic Society funded fieldwork in 1993 near the proposed park boundaries that was essential to our calculations. Alan Perry and Tom Schulenberg provided valuable comments on the manuscript.

\section{References}

Collar, N. J., Gonzaga, L. P., Krabbe, N., Madroño Nieto, A., Naranjo, L. G., Parker, T. A. and Wege, D. C. (1992) Threatened birds of the Americas: the ICBP/IUCN Red Data Book. Cambridge, U.K.: International Council for Bird Preservation.

Fjeldså, J. and Krabbe, N. (1990) Birds of the high Andes. [Copenhagen:] Zoological Museum, University of Copenhagen, and Svendborg: Apollo Books.

Fjeldså, J. (1992) Biogeographic patterns and evolution of the avifauna of relict high altitude woodlands of the Andes. Streenstrupia 18: 9-62.

Foster, R. B., Parker T. A., III, Gentry, A. H., Emmons, L. H., Chicchón, A., Schulenberg, T. S., Rodríguez, L., Lamas, G., Ortega, H., Icochea, J., Wust, W., Romo, M., Castillo, A. A., Phillips, O., Reynel, C., Kratter, A. W., Donahue, P. K. and Barkley, L. J. (1994) The Tambopata-Candamo Reserved Zone of southeastern Peri: a biological assessment. Washington, D.C.: Conservation International (Rapid Assessment Program Working Pap. 6).

Graham, G. L., Graves, G. R., Schulenberg, T. S. and $O^{\prime}$ Neill, J. P. (1980) Seventeen bird species new to Peru from the Pampas de Heath. Auk 97: 366-370.

Parker, T. A. and Bailey, B. (1991) A biological assessment of the Alto Madidi region and adjacent areas of northwest Bolivia, May 18-June 15, 1990. Washington, D.C.: Conservation International (Rapid Assessment Program Working Pap. 1).

Parker, T. A. and Remsen, J. V. (1987) Fifty-two Amazonian bird species new to Bolivia. Bull. Brit. Orn. Club 107: 94-107. 
Parker, T. A., Castillo U., A., Gell-Mann, M. and Rocha O., O. (1991) Records of new and unusual birds from northern Bolivia. Bull. Brit. Orn. Club 111: 120-138.

Pearman, M. (1993) The avifauna of the Río Machariapo dry forest, northern La Paz department, Bolivia: a preliminary investigation. Bird Conserv. Internatn. 3: 105-117.

Remsen, J. V., Jr. (1984) Geographic variation, zoogeography, and possible rapid evolution in some Cranioleuca spinetails. Wilson Bull. 96: 515-523.

Remsen, J. V., Jr., and Traylor, M. A., Jr. (1989) An annotated list of the birds of Bolivia. Vermillion, South Dakota: Buteo Books.

Ribera, M. O. and Hanagarth, W. (1982) Aves de la region altoandina de La Reserva Nacional de Ulla-Ulla. Ecologia en Bolivia 1: 35-45.

Whitney, B. M. (1994) A new Scytalopus tapaculo (Rhinocryptidae) from Bolivia, with notes on other Bolivian members of the genus and the magellanicus complex. Wilson Bull. 106: 585-614.

J. V. REMSEN, JR. and T. A. PARKER, III

Museum of Natural Science, Louisiana State University, Baton Rouge, Louisiana 70803, U.S.A.

\section{Appendix. Bird species recorded or predicted to occur in proposed park or reserve system, La Paz, Bolivia.}

$X$ indicates that the species has already been recorded. Numbers refer to the following sources: 1, Parker and Bailey (1991); 2, Ribera and Hanagarth (1982); 3. Pearman (1993); 4, T. A. Parker's survey from the Bolivian side of Río Heath (in Remsen and Traylor 1989, Parker et al. 1991), only included for species not recorded elsewhere in region; 5, Whitney (1994). Expected species are indicated by $E$, and species that might possibly be present by $P$ (see text for criteria). Species with small geographical ranges (see Methods) are noted by an asterisk $\left({ }^{*}\right)$ after the name. Migrants from the Nearctic region are indicated by BM (boreal migrant) after the name. Because knowledge of seasonal movements of many South American species is limited, we do not designate suspected austral or intratropical migrants.

\begin{tabular}{|c|c|c|c|}
\hline RHEIDAE (2 species) & & Crypturellus variegatus & $X(1)$ \\
\hline Rhea americana & $\mathrm{P}$ & Crypturellus bartletti & $X(1)$ \\
\hline Pterocnemia pennata & $\mathrm{P}$ & Crypturellus parvirostris & $\mathrm{E}$ \\
\hline TINAMIDAE (2I species) & & Crypturellus tataupa & $X(3)$ \\
\hline Tinamus tao & $X(1,3)$ & Rhynchotus rufescens & $\mathrm{E}$ \\
\hline Tinamus major & $X(1)$ & Nothoprocta ornata & $\mathrm{E}$ \\
\hline Tinamus osgoodi & $\mathrm{E}$ & Nothoprocta pentiandii & $\mathrm{E}$ \\
\hline Tinamus guttatus & $X(1)$ & Nothoprocta taczanowskii & $\mathrm{E}$ \\
\hline Nothocercus nigrocapillus & $\mathrm{E}$ & Nothura darwinii & E \\
\hline Crypturellus cinereus & $X(1)$ & Inamotis pentlandn & $X(2)$ \\
\hline Crypturellus soui & $X(1)$ & PODICIPEDIDAE (4 species) & \\
\hline Crypturellus obsoletus & $X(1)$ & Rollandia rolland & $X(2)$ \\
\hline Crypturellus undulatus & $X(1,3)$ & Tachybaptus dominicus & $\mathrm{E}$ \\
\hline Crypturellus strigulosus & $\mathrm{E}$ & Podilymbus podiceps & $\mathrm{P}$ \\
\hline Crypturellus atrocapillus & $X(3)$ & Podiceps occipitalis & $X(2)$ \\
\hline
\end{tabular}


PHALACROCORACIDAE (1 species) ANHIMIDAE (2 species)

Phalacrocorax brasilianus

ANHINGIDAE (1 species)

Anhinga anhinga

ARDEIDAE (17 species)

Ixobrychus involucris

Ixobrychus exilis

Botaurus pinnatus

Zebrilus undulatus

Tigrisoma fasciatum

Tigrisoma lineatum

Syrigma sibilatrix

Pilherodius pileatus

Ardea cocoi

Ardea alba

Bubulcus ibis

Egretta thula

Egretta caerulea

Butorides striatus

Agamia agami

Nycticorax nycticorax

Cochlearius cochlearius
$X(1,2)$

Anhima cornuta

Chauna torquata

$X(1)$

E

E

E

E

E

$X(1)$

E

$X(1)$

$X(1)$

$X(1,2)$

$X(1)$

$X(1)$

$\mathrm{E}$

$X(1)$

E

$X(1,2)$

$\mathrm{E}$

Neochen jubata

Anas flavirostris

Anas georgica

Anas bahamensis

Anas versicolor

Anas cyanoptera

Anas platalea

Oxyura dominica
PHOENICOPTERIDAE (3 species)

Phoenicopterus chilensis

Phoenicoparrus andinus

Phoenicoparrus jamesi

THRESKIORNITHIDAE (7 species)

Phimosus infuscatus

Plegadis ridgwayi

Theristicus caerulescens

Theristicus caudatus

Theristicus melanopis

Mesembrinibis cayennensis

Platalea ajaja

\section{CICONIIDAE (3 species)}

Mycteria americana

Ciconia maguari

Jabiru mycteria

\section{CATHARTIDAE (6 species)}

Coragyps atratus

Cathartes aura

Cathartes burrovianus

Cathartes melambrotus

Vultur gryphus

Sarcoramphus papa
$X(2)$

$\mathrm{P}$

$\mathrm{P}$

$\mathrm{P}$

$X(2)$

$\mathrm{E}$

E

$\mathrm{X}(2) \quad$ Ictinia missisippiensis (BM)

$X(1)$

$X$

$X(1)$

$P$

$X(1)$

$X(1,3)$

$X(3)$

$\mathrm{E}$

$X(1)$

$\mathrm{E}$

$X(1)$

Leptodon cayanensis

Chondrohierax uncinatus

Elanoides forficatus

Gampsonyx swainsonii

Elanus caeruleus

Rostrhamus sociabilis
ANATIDAE (18 species)

Dendrocygna bicolor

Dendrocygna viduata

Dendrocygna autumnalis

Chloephaga melanoptera

Cairina moschata

Callonetta leucophrys

Amazonetta brasiliensis

Merganetta armata

Anas specularioides

Oxyura jamaicensis

$X(1)$

$X(1)$

E

E

$\mathrm{E}$

$X(2)$

$X(1)$

$X(1)$

E

E

E

$X(2)$

$X(2)$

$X(2)$

$\mathrm{E}$

$X(2)$

$\mathrm{E}$

$\mathrm{P}$

$\mathrm{E}$

$X(2)$

ACCIPITRIDAE (44 species)

Pandion haliaetus

$X(1)$

$X(1)$

$\mathrm{E}$

$X(1)$

$X(1)$

E

E

E

$X(1)$

$\mathrm{P}$

E

E

E

E

E

$\mathrm{E}$

$X(1)$

$X(1)$

$X(1)$

$\mathrm{E}$

$X(1)$

$X(1)$

$X(I)$

E 
Parabuteo unicinctus

Busarellus nigricollis

Geranoaetus melanoleucus

Harpyhaliaetus solitarius

Buteo magnirostris

Buteo leucorrhous

Buteo platypterus (BM)

Buteo brachyurus

Buteo albigula

Buteo swainsonii (BM)

Buteo albicaudatus

Buteo polyosoma

Buteo poecilochrous

Buteo albonotatus

Morphnus guianensis

Harpia harpyja

Spizastur melanoleucus

Spizaetus tyrannus

Spizaetus ornatus

Oroaetus isidori

\section{FALCONIDAE (15 species)}

Daptrius ater

Daptrius americanus

Phalcoboenus megalopterus

Polyborus plancus

Milvago chimachima

Herpetotheres cachinnans

Micrastur ruficollis

Micrastur gilvicollis

Micrastur mirandollei

Micrastur semitorquatus

Falco sparverius

Falco femoralis

Falco rufigularis

Falco peregrinus

Falco deiroleucus

\section{CRACIDAE (9 species)}

Ortalis motmot

Penelope montagnii

Penelope jacquacu

Pipile cumanensis

Chamaepetes goudotii

Mitu tuberosa

Pauxi unicornis

Crax globulosa

Crax fasciolata
E

E

E

E

$X(1,3)$

E

E

E

E

E

$\mathrm{E}$

E

$X(2)$

$E$

$X(1)$

E

E

$X(1)$

$X(4)$

E

$X(1)$
$X(1)$
$X(2)$
$E$
$E$
$X(1)$
$X(1,3)$
$X(1)$
$E$
$E$
$X$
$E$
$X(1)$
$E$
$E$

$X(1,3)$

$\mathrm{E}$

$X(1)$

$X(1)$

E

$X(1)$

$\mathrm{E}$

$P$

E
ODONTOPHORIDAE (4 species)

Odontophorus gujanensis

$\mathrm{E}$

Odontophorus speciosus

E

$\mathrm{E}$

$X(1)$

Odontophorus ballivian

Odontophorus stellatus

RALLIDAE (17 species)

Rallus sanguinolentus

$E$

Rallus nigricans

$X(1)$

Pardirallus maculatus

E

E

$X(1)$

$\mathrm{E}$

E

E

$X(1)$

$X(1)$

$\mathrm{E}$

$\mathrm{E}$

$X(2)$

$X(1)$

$\begin{array}{ll}\text { Porphyrula flavirostris } & \text { E } \\ \text { Fulica ardesiaca } & \mathrm{X}(2) \\ \text { Fulica gigantea } & \mathrm{X}(2)\end{array}$

$\begin{array}{ll}\text { Porphyrula flavirostris } & \mathrm{E} \\ \text { Fulica ardesiaca } & \mathrm{X}(2) \\ \text { Fulica gigantea } & \mathrm{X}(2)\end{array}$

$\begin{array}{ll}\text { Porphyrula flavirostris } & \text { E } \\ \text { Fulica ardesiaca } & \mathrm{X}(2) \\ \text { Fulica gigantea } & \mathrm{X}(2)\end{array}$

HELIORNITHIDAE (1 species)

Heliornis fulica

$X$

EURYPYGIDAE (1 species)

Eurypyga helias

$X(1)$

ARAMIDAE (1 species)

Aramus guarauna

E

PSOPHIIDAE (1 species)

Psophia leucoptera

$X(1)$

CHARADRIIDAE (8 species)

Vanellus cayanus

$X(1)$

Vanellus chilensis

$\mathrm{E}$

Vanellus resplendens $\quad X(2)$

$X(2)$

Pluvialis dominicus (BM)

$X(1)$

Charadrius collaris

$\mathrm{E}$

$\mathrm{E}$

$\mathrm{E}$

Eudromias ruficollis

RECURVIROSTRIDAE (2 species)

Himantopus mexicanus

E

Recurvirostra andina

$X(2)$ 
JACANIDAE (1 species)

Jacana jacana

\section{SCOLOPACIDAE (14 species)}

Bartramia longicauda (BM)

Tringa melanoleuca (BM)

Tringa flavipes (BM)

Tringa solitaria (BM)

Actitis macularia (BM)

Phalaropus tricolor (BM)

Gallinago paraguaiae

Gallinago jamesoni

Calidris minutilla (BM)

Calidris fuscicollis (BM)

Calidris bairdii (BM)

Calidris melanotos (BM)

Calidris himantopus (BM)

Tryngites subruficollis (BM)

THINOCORIDAE (3 species)

Attagis gayi

Thinocorus orbignyiantus

Thinocorus rumicioorus

\section{LARIDAE ( 3 species)}

Larus serranus

Phaetusa simplex

Sterna superciliaris

\section{RYNCHOPIDAE (1 species)}

Rynchops niger

COLUMBIDAE (23 species)

Columba speciosa

Columba maculosa

Columba fasciata

Columba cayennensis

Columba plumbea

Columba subvinacea

Zenaida auriculata

Columbina minuta

Columbina talpacoti

Columbina picui

Claravis pretiosa

Claravis mondetoura

Metriopelia ceciliae

Metriopelia melanoptera

Metriopelia aymara

Uropelia campestris

Leptotila verreauxi
E

$\mathrm{E}$

E

$X(2)$

$X(4) \quad$ PSITTACIDAE (34 species)

E Ara ararauna

$\mathrm{X}(2) \quad$ Ara militaris

$\mathrm{E} \quad$ Ara macao

E Ara chloroptera

$\mathrm{P} \quad$ Ara severa

E Ara manilata

X(2) Ara couloni*

$\mathrm{E} \quad$ Ara nobilis

E Aratinga leucophthalmus

E Aratinga weddellit

Aratinga aurea

Pyrrhura molinae

E Pyrrhura picta

X(2) Pyrrhura rupicola

$\mathrm{E}$

Bolborhynchus aurifrons

Bolborhynchus orbygnesius

$X(2) \quad$ Forpus xanthopterygius

$X(1) \quad$ Forpus sclateri

$X(1) \quad B r o t o g e r i s$ versicolurus

Brotogeris cyanoptera

Brotogeris sanctithomae

$X(1)$

\section{E}

P

E

$X(1)$

$\mathrm{E}$

$X(1)$

$X(3)$

$\mathrm{E}$

$X(1)$

$X(1,3)$

$X(1)$

$\mathrm{E}$

$\mathrm{E}$

$X(2)$

$\mathrm{E}$

$\mathrm{E}$

$X(3)$

Nannopsittaca dachilleae
$\mathrm{E}$

$X(1)$

$\mathrm{E}$

E

E

$X(1)$

$X(1)$

E

$X(1)$

$X(1)$

$X(1)$

$X(1)$

E

$X(4)$

$X(1,3)$

$X(1)$

$X(4)$

E

$X(1)$

$X(1)$

P

P

E

$X(1)$

E

$X(1)$

E

$X(1)$

$X(4)$

$X(1)$

$X(1)$

E

$X(1,3)$

E

E

E

$X(1)$

E

E

$X(1)$

E

$X(3)$

E

$X(1)$

$X(1,3)$ 


\begin{tabular}{|c|c|c|c|}
\hline Piaya melanogaster & $X(1)$ & Chordeiles acutipennis & E \\
\hline Piaya minuta & $X(1)$ & Podager nacunda & $\mathrm{E}$ \\
\hline Crotophaga major & $\mathrm{E}$ & Nyctidromus albicollis & $X(1,3)$ \\
\hline Crotophaga ani & $X(1)$ & Nyctiphrynus ocellatus & $X(1)$ \\
\hline Guira guira & $\mathrm{E}$ & Caprimulgus sericocaudatus & $\mathrm{E}$ \\
\hline Tapera naevia & $X(1)$ & Caprimulgus longirostris & $\mathrm{E}$ \\
\hline Dromococcyx phasianell & $X(1)$ & Caprimulgus maculicaudus & $\mathrm{E}$ \\
\hline Dromococcyx pavoninus & $X(1)$ & Caprimulgus paroulus & $\mathrm{E}$ \\
\hline Neomorphus geoffroyi & $X(3)$ & Hydropsalis climacocerca & $X(1)$ \\
\hline \multicolumn{2}{|c|}{ OPISTHOCOMIDAE (1 species) } & Hydropsalis brasiliana & $\mathrm{E}$ \\
\hline \multirow{2}{*}{ Opisthocomus hoazin } & \multirow[t]{2}{*}{$X(1)$} & Uropsalis segmentata & $\mathrm{E}$ \\
\hline & & Uropsalis lyra & $\mathrm{E}$ \\
\hline \multirow{2}{*}{$\begin{array}{l}\text { TYTONIDAE (1 species } \\
\text { Tyto alba }\end{array}$} & \multirow{2}{*}{$\mathrm{E}$} & APODIDAE (11 species) & \\
\hline & & Cypseloides cryptus & $\mathrm{E}$ \\
\hline \multicolumn{2}{|c|}{ STRIGIDAE (18 species) } & Streptoprocne rutila & $\mathrm{E}$ \\
\hline Otus guatemalae & $X(3)$ & Streptoprocne zonaris & $X(1)$ \\
\hline Otus choliba & $X(1)$ & Chaetura chapmani & $\mathrm{E}$ \\
\hline Otus ingens & $\mathrm{E}$ & Chaetura cinereiventris & $X(1)$ \\
\hline Otus watsonii & $X(1)$ & Chaetura egregia & $X(1)$ \\
\hline Otus albogularis & $\mathrm{E}$ & Chaetura andrei & $\mathrm{E}$ \\
\hline Lophostrix cristata & $X(1)$ & Chaetura brachyura & $X(1)$ \\
\hline Bubo virginianus & $\mathrm{E}$ & Aeronautes montivagus & $\mathrm{E}$ \\
\hline Pulsatrix perspicillata & $X(1)$ & Panyptila cayennensis & $X(1)$ \\
\hline Pulsatrix melanota & $\mathrm{E}$ & Tachornis squamata & $X(4)$ \\
\hline \multirow{2}{*}{$\begin{array}{l}\text { Glaucidium hardyi } \\
\text { Glaucidium bolivianum }\end{array}$} & $X(1)$ & \multirow{2}{*}{\multicolumn{2}{|c|}{ TROCHILIDAE (61 species) }} \\
\hline & $X(1)$ & Doryfera ludoviciae. & \\
\hline Glaucidium brasilianum & $X(1)$ & & \\
\hline Speotyto cunicularia & $\mathrm{E}$ & Glaucis hirsuta & $X(1)$ \\
\hline Ciccaba virgata & $X(\mathbf{1})$ & Threnetes leucurus & $X(1)$ \\
\hline Ciccaba huhula & $\mathrm{E}$ & Phaethornis superciliosus & $X(1)$ \\
\hline Ciccaba albitarsus & $\mathrm{E}$ & Phaethornis hispidus & $X(1)$ \\
\hline Rhinoptynx clamator & $\mathrm{E}$ & Phaethornis philippii & $X(4)$ \\
\hline Asio flammeus & $\mathrm{E}$ & Phaethornis stuarti* & $E$ \\
\hline & & Phaethornis ruber & $X(1)$ \\
\hline \multicolumn{2}{|c|}{ STEATORNITHIDAE (1 species) } & Eutoxeres condamini & $\mathrm{E}$ \\
\hline Steatornis caripensis & $\mathrm{E}$ & Campylopterus largipennis & $\mathrm{E}$ \\
\hline \multicolumn{2}{|c|}{ NYCTIBIIDAE (5 species) } & Eupetomena macroura & $\mathrm{E}$ \\
\hline Nyctibius grandis & $X(1)$ & Florisuga mellivora & $X(1)$ \\
\hline Nyctibius griseus & $\mathrm{E}$ & Colibri delphinae & $\mathrm{E}$ \\
\hline Nyctibius maculosus & $\mathrm{E}$ & Colibri thalassinus & $\mathrm{E}$ \\
\hline Nyctibius aethereus & $\bar{E}$ & Colibri coruscans & $\mathrm{E}$ \\
\hline Nyctibius bracteatus & $\mathrm{E}$ & Anthracothorax nigricollis & $X(1)$ \\
\hline \multirow{2}{*}{\multicolumn{2}{|c|}{ CAPRIMULGIDAE (16 species) }} & Klais guimeti & $X(3)$ \\
\hline & & Lophornis delattrei & $\mathrm{E}$ \\
\hline Lurocalis semitorquatus & $\mathrm{E}$ & Lophornis chalybea & E \\
\hline Lurocalis rufiventris & $\mathrm{E}$ & Popelairia langsdorffi & $\mathrm{E}$ \\
\hline Chordeiles rupestris & $X(1)$ & Popelairia letitiae* & $\mathrm{P}$ \\
\hline Chordeiles minor (BM) & $\mathrm{E}$ & Chlorostilbon mellisugus & $\mathrm{E}$ \\
\hline
\end{tabular}


Thalurania furcata

Hylocharis sapphirina

Hylocharis cyanus

Chrysuronia oenone

Polytmus guainumbi

Polytmus theresiae

Calliphlox amethystina

Taphrospilus hypostictus

Amazilia fimbriata

Amazilia lactea

Adelomyia melanogenys

Polyplancta aurescens

Heliodoxa leadbeateri

Oreotrochilus estella

Patagona gigas

Aglaeactis pamela*

Pterophanes cyanoptera

Coeligena coeligena

Coeligena torquata

Coeligena violifer

Ensifera ensifera

Heliangelus amethysticollis

Eriocnemis luciani

Haplophaedia aureliae

Ocreatus underwoodii

Lesbia nuna

Ramphomicron microrhynchum

Metallura aeneocauda

Metallura tyrianthina

Chalcostigma ruficeps

Chalcostigma olivaceum

Chalcostigma stanleyi

Aglaiocercus kingi

Schistes geoffroyi

Heliothryx aurita

Heliomaster longirostris

Heliomaster furcifer

Calliphlox amethystina

Acestrura mulsant

TROGONIDAE (9 species)

Pharomachrus antisianus

Pharomachrus auriceps

Pharomachrus pavoninus

Trogon melanurus

Trogon viridis

Trogon collaris

Trogon personatus

$\begin{array}{lll}\mathrm{X}(1) & \text { Trogon curucui } & \mathrm{X}(1,3) \\ \mathrm{P} & \text { Trogon violaceus } & \mathrm{X}(1) \\ \mathrm{X}(1) & \text { MOMOTIDAE (3 species) } & \\ \mathrm{E} & \text { Electron platyrhynchum } & \mathrm{X}(1) \\ \mathrm{E} & \text { Baryphthengus martii } & \mathrm{X}(1) \\ \mathrm{E} & \text { Momotus momota } & \mathrm{X}(1,3) \\ \mathrm{E} & & \\ \mathrm{E} & \text { ALCEDINIDAE (5 species) } & \\ \mathrm{E} & \text { Ceryle torquata } & \mathrm{X}(1) \\ \mathrm{X}(1) & \text { Chloroceryle amazona } & \mathrm{X}(1) \\ \mathrm{X} & \text { Chloroceryle americana } & \mathrm{X}(1) \\ \mathrm{X}(1) & \text { Chloroceryle inda } & \mathrm{X}(1) \\ \mathrm{X} & \text { Chloroceryle aenea } & \mathrm{X}(1) \\ \mathrm{E} & \text { BUCCONIDAE (15 species) } & \\ \mathrm{E} & \text { Notharchus macrorhynchos } & \mathrm{X}(1) \\ \mathrm{E} & \text { Notharchus ordii } & \mathrm{E} \\ \mathrm{E} & \text { Notharchus tectus } & \mathrm{P} \\ \mathrm{X} & \text { Bucco macrodactylus } & \mathrm{E} \\ \mathrm{E} & \text { Bucco capensis } & \mathrm{E} \\ \mathrm{E} & \text { Nystalus chacuru } & \mathrm{X}(3) \\ \mathrm{E} & \text { Nystalus striolatus } & \mathrm{X}(1) \\ \mathrm{E} & \text { Malacoptila semicincta } & \mathrm{X}(1) \\ \mathrm{E} & \text { Malacoptila fulvogularis } & \mathrm{E} \\ \mathrm{X} & \text { Nonnula sclateri } & \mathrm{E} \\ \mathrm{X} & \text { Nonnula ruficapilla } & \mathrm{X}(1) \\ \mathrm{E} & \text { Monasa nigrifrons } & \mathrm{X}(1) \\ \mathrm{P} & \text { Monasa morphoeus } & \mathrm{X}(1) \\ \mathrm{E} & \text { Monasa flavirostris } & \mathrm{E} \\ \mathrm{E} & \text { Chelidoptera tenebrosa } & \mathrm{X}(1) \\ \mathrm{E} & \text { GALBULIDAE (7 species) } & \\ \mathrm{E} & \text { Galbalcyrhynchus purusianus } & \mathrm{E} \\ \mathrm{E} & \text { Brachygalba albogularis } & \mathrm{X}(4) \\ \mathrm{X} & \mathrm{X}(1) \\ \mathrm{E} & \text { Galbula cyanescens } & \mathrm{X}(3) \\ \mathrm{X}(1) & \text { Galbula ruficauda } & \mathrm{P} \\ \mathrm{X}(1) & \text { Galbula leucogastra } & \mathrm{X}(4) \\ \mathrm{P} & \text { Galbula dea } & \mathrm{X}(1) \\ \mathrm{E} & \text { Jacamerops aurea } & \\ \mathrm{X} & & \\ & \text { CAPITONIDAE (4 spe } & \end{array}$

CAPITONIDAE (4 species)

Capito niger

$X(1)$

$X(1)$

E

$\mathrm{X}(1) \quad$ Eubucco tucinckae $\mathrm{X}(\mathrm{I})$

$X(1)$

$X(1)$ RAMPHASTIDAE (12 species)

$\mathrm{X}(\mathrm{1}) \quad$ Aulacorhynchus prasinus $\mathrm{X}(1)$

E Aulacorhynchus derbianus E 


Aulacorhynchus coeruleicinctis
Pteroglossus inscriptus
Pteroglossus flavirostris
Pteroglossus castanotis
Pteroglossus beauharnaesii
Selenidera reinwardtii
Andigena cucullata*
Ramphastos vitellinus
Ramphastos tucanus
Ramphastos toco

\section{PICIDAE (24 species)}

Picumnus rufioentris

Picumnus minutissimus

Picumnus aurifrons

Melanerpes candidus

Melanerpes cruentatus

Veniliornis nigriceps

Veniliornis fumigatus

Veniliornis passerinus

Veniliornis affinis

Piculus leticolaemus

Piculus chrysochloros

Piculus rubiginosus

Piculus rivolii

Colaptes punctigula

Colaptes rupicola

Colaptes campestris

Celeus grammicus

Celeus elegans

Celeus flavus

Celeus spectabilis

Celeus torquatus

Dryocopus lineatus

Campephilus melanoleucos

Campephilus rubricollis

\section{DENDROCOLAPTIDAE (21 species)}

Dendrocincla fuliginosa

Dendrocincla merula

Deconychura longicauda

Sittasomus griseicapillus

Glyphorynchus spirurus

Nasica longirostris

Dendrexetastes rufigula

Hylexetastes stresemanni

Xiphocolaptes promeropirhynchus

Dendrocolaptes certhia
E

X(1) Xiphorhynchus picus

$X(1) \quad X i p h o r h y n c h u s$ obsoletus

$X(1,3) \quad X i p h o r h y n c h u s$ ocellatus

$X(1) \quad$ Xiphorhynchus spixii

$X(1) \quad X i p h o r h y n c h u s$ guttatus

E Xiphorhynchus triangularis

$X(1) \quad$ Lepidocolaptes angustirostris

$X(1)$

E

Lepidocolaptes affinis

Lepidocolaptes albolineatus

Campylorhamphus trochilirostris

\section{E FURNARIIDAE (74 species)}

$X(3) \quad$ Geositta punensis

$X(1) \quad$ Geositta cunicularia

E Geositta tenuirostris

$\mathrm{X}(\mathrm{1}) \quad$ Upucerthia dumetaria

$\mathrm{E}$

E

$X(1)$

$X(1,3)$

$X(1)$

$X(1)$

E

$\mathrm{E}$

$X(1)$

$X(2)$

E

$X(1)$

$X(1)$

$X(1)$

$X(1)$

$X(1)$

$X(1,3)$

$X(1,3)$

$X(1)$

Upucerthia jelskii

Upucerthia ruficauda

Cinclodes aricomae

Cinclodes fuscus

Cinclodes atacamensis

Furnarius rufus

Furnarius leucopus

Phleocryptes melonops

Leptasthenura fuliginiceps

Leptasthenura yanacensis

Schoeniophylax phryganophila

Synallaxis azarae

Synallaxis frontalis

Synallaxis cabanisi

Synallaxis hypospodia

Synallaxis albescens

Synallaxis gujanesis

Synallaxis rutilans

Certhiaxis cinnamomea

Poecilurus scutatus

Cranioleuca curtata

$X(1) \quad$ Cranioleuca vulpina

$X(1)$

Cranioleuca albiceps*

$X(1)$

Cranioleuca gutturata

$X(1,3) \quad$ Schizoeaca helleri

X(1) Asthenes dorbignyi

X(4) Asthenes berlepschi*

$X(1)$ Asthenes modesta

$\mathrm{P}$

Asthenes heterura*

Asthenes wyatti

Asthenes humilis
$X(1,3)$

$X(1)$

$X(4)$

$\mathrm{E}$

$X(1)$

$X(1)$

$\mathrm{E}$

E

$X(3)$

$X(1)$

$X(1,3)$
E

$X(2)$

$E$

$X(2)$

E

P

$\mathrm{E}$

$X(2)$

$X(2)$

E

$X(1)$

E

P

E

E

E

E

E

$\mathrm{E}$

$X(1)$

$X(1)$

$X(1)$

$E$

$X(3)$

$\mathrm{E}$

E

E

$X(1)$

$X(5)$

E

E

E

E

E

E 


\begin{tabular}{|c|c|c|c|}
\hline Asthenes maculicauda & $\mathrm{E}$ & Thamnophilus schistaceus & $X(1)$ \\
\hline Asthenes urubambensis & $\mathrm{E}$ & Thamnophilus aroyae ${ }^{*}$ & $X(3)$ \\
\hline Thripophaga fusciceps & $X(4)$ & Thamnophilus amazonicus & $\mathrm{E}$ \\
\hline Phacellodomus striaticeps & $\mathrm{E}$ & Thamnophilus caerulescens & $\mathrm{E}$ \\
\hline Phacellodomus ruber & $X(3)$ & Thamnophilus ruficapillus & $\mathrm{E}$ \\
\hline Metopothrix aurantiacus & $\mathrm{E}$ & Pygiptila stellaris & $X(1)$ \\
\hline Margarornis squamiger & $\mathrm{E}$ & Thamnistes anabatinus & $\mathrm{E}$ \\
\hline Premnoplex brunnescens & $\mathrm{E}$ & Dysithammus mentalis & $X(3)$ \\
\hline Premnornis guttuligera & $\mathrm{E}$ & Thamnomanes ardesiacus & $X(1)$ \\
\hline Pseudocolaptes boissonneautii & $\mathrm{E}$ & Thamnomanes schistogynus & $X(1)$ \\
\hline Berlepschia rikeri & $\mathrm{E}$ & Myrmotherula brachyura & $X(1)$ \\
\hline Hyloctistes subulatus & $X(1)$ & Myrmotherula sclateri & $X(1)$ \\
\hline Ancistrops strigilatus & $X(1)$ & Myrmotherula surinamensis & $X(4)$ \\
\hline Syndactyla rufosuperciliata & $\mathrm{E}$ & Myrmotherula longicauda & $X(3)$ \\
\hline Simoxenops ucayalae & $\mathrm{E}$ & Myrmotherula hauxwelli & $X(1)$ \\
\hline Simoxenops striatus* & $P$ & Myrmotherula leucophthalma & $X(1)$ \\
\hline Anabacerthia striaticollis & E & Myrmotherula haematonota & $\mathrm{E}$ \\
\hline Philydor erythrocercus & $X(1)$ & Myrmotherula ornata & $\mathrm{E}$ \\
\hline Philydor pyrrhodes & $X(1)$ & Myrmotherula erythrura & $\mathrm{E}$ \\
\hline Philydor rufus & $X(1)$ & Myrmotherula axillaris & $X(1)$ \\
\hline Philydor erythropterus & $X(1)$ & Myrmotherula longipennis & $X(1)$ \\
\hline Philydor ruficaudatus & $X$ & Myrmotherula iheringi & $\mathrm{P}$ \\
\hline Anabazenops dorsalis & $X(1)$ & Myrmotherula grisea* & $\mathrm{E}$ \\
\hline Automolus infuscatus & $X(1)$ & Myrmotherula menetriesii & $X(1)$ \\
\hline Automolus rubiginosus & $\mathrm{E}$ & Dichrozona cincta & $X(1)$ \\
\hline Automolus ochrolaemus & $X(1)$ & Herpsilochmus sp. nov. ${ }^{*}$ & $X(1,3)$ \\
\hline Automolus rufipileatus & $X(1)$ & Herpsilochmus rufimarginatus & $\mathrm{E}$ \\
\hline Automolus melanopezus & $\mathrm{E}$ & Microrhopias quixensis & $\mathrm{E}$ \\
\hline Thripadectes holostictus & $X$ & Formicivora melanogaster & $X(3)$ \\
\hline Thripadectes scrutator & $\mathrm{E}$ & Formicioora rufa & $\mathrm{E}$ \\
\hline Xenops milleri & $X(1)$ & Drymophila devillei & $\mathrm{E}$ \\
\hline Xenops tenuirostris & $\mathrm{E}$ & Drymophila caudata & $\mathrm{E}$ \\
\hline Xenops rutilans & $X(1)$ & Terenura humeralis & $X(1)$ \\
\hline Xenops minutus & $X(1,3)$ & Terenura sharpei* & $\mathrm{E}$ \\
\hline Sclerurus albigularis & $\mathrm{E}$ & Cercomacra cinerascens & $X(1)$ \\
\hline Sclerurus mexicanus & E & Cercomacra nigrescens & $X(1)$ \\
\hline Sclerurus rufigularis & $\mathrm{E}$ & Cercomacra serva & $X(1)$ \\
\hline Sclerurus caudacutus & $X(1)$ & Cercomacra manu & $X(1)$ \\
\hline Lochmias nematura & $\mathrm{E}$ & Cercomacra melanaria & $\mathrm{P}$ \\
\hline & & Pyriglena leuconota & $X(3)$ \\
\hline \multicolumn{2}{|c|}{ FORMICARIIDAE (83 species) } & Neoctantes niger & $\mathrm{P}$ \\
\hline Cymbilaimus lineatus & $X(1)$ & Myrmoborus leucophrys & $X(1)$ \\
\hline Cymbilaimus sanctaemariae ${ }^{*}$ & $X(4)$ & Myrmoborus myotherinus & $X(1)$ \\
\hline Frederickena unduligera & $X(1)$ & Hypocnemis cantator & $X(1)$ \\
\hline Taraba major & $X(1,3)$ & Hypocnemoides maculicauda & $E$ \\
\hline Thamnophilus doliatus & $X(1)$ & Sclateria naevia & $X(1)$ \\
\hline Thamnophilus palliatus & $X(3)$ & Schistocichla leucostigma & $\mathrm{E}$ \\
\hline Thamnophilus aethiops & $X(1)$ & Percnostola lophotes* & $X(1)$ \\
\hline
\end{tabular}


Myrmeciza hemimelaena

Myrmeciza hyperythra

Myrmeciza goeldii*

Myrmeciza fortis

Myrmeciza atrothorax

Gymnopithys salvini

Rhegmatorhina melanosticta

Hylophylax naevia

Hylophylax punctulata

Hylophylax poecilinota

Phlegopsis nigromaculata

Phlegopsis erythroptera

Formicarius colma

Formicarius analis

Formicarius rufifrons*

Chamaeza nobilis

Chamaeza campanisona

Chamaeza mollissima

Grallaria squamigera

Grallaria guatimalensis

Grallaria albigula

Grallaria rufula

Grallaria erythrotis*

Hylopezus macularius

Hylopezus berlepschi

Myrmothera campanisona

Grallaricula flavirostris

Grallaricula ferrugineipectus

\section{CONOPOPHAGIDAE (2 species)}

Conopophaga peruviana

Conopophaga ardesiaca

\section{RHINOCRYPTIDAE (4 species)}

Scytalopus parvirostris

Scytalopus bolivianus

Scytalopus schulenbergi*

Scytalopus magellanicus

\section{TYRANNIDAE (191 species)}

Phyllomyias burmeisteri

Phyllomyias sclateri

Phyllomyias cinereiceps

Phyllomyias uropygialis

Zimmerius bolivianus

Zimmerius gracilipes

Ornithion inerme

Camptostoma obsoletum

Phaeomyias murina
$X(1) \quad$ Sublegatus modestus

$X(1) \quad$ Sublegatus obscurior

E

$X(1)$

$X(1) \quad$ Suiriri suiriri

$X(1) \quad$ Tyrannulus elatus

$X(1) \quad$ Myiopagis gaimardii

$\mathrm{E}$

$X(1)$

$X(1)$

$X(1)$

$X(1,3)$

$\mathrm{E}$

$X(1)$

$X(3)$

$X(4)$

$\mathrm{E}$

E

E

E

E

E

E

E

$\mathrm{E}$

E

E

$X(1)$

$P$

$\mathrm{P}$

E

$\mathrm{P}$

E

P

E

$X(1)$

$X(1)$

$\mathrm{E}$

$X(1)$

$X(1)$

$X(1,3)$

$\mathrm{E}$

$\mathrm{E}$

E

E

E

$\mathrm{E}$

E

$X(1,3)$

$\mathrm{E}$

$\mathrm{E}$

$X(3)$

$X(1)$ 
Lophotriccus eulophotes

Hemitriccus flammulatus

Hemitriccus zosterops

Hemitriccus iohannis

Hemitriccus striaticollis

Hemitriccus spodiops*

Hemitriccus margaritaceiventer

Hemitriccus granadensis

Hemitriccus rufigularis

Poecilotriccus albifacies ${ }^{*}$

Todirostrum plumbeiceps

Todirostrum latirostre

Todirostrum maculatum

Todirostrum cinereum

Todirostrum chrysocrotaphum

Cnipodectes subbrunneus

Ramphotrigon megacephala

Ramphotrigon fuscicauda

Ramphotrigon ruficauda

Rhynchocyclus olivaceus

Rhynchocyclus fulvipectus

Tolmomyias sulphurescens

Tolmomyias assimilis

Tolmomyias poliocephalus

Tolmomyias flaviventris

Platyrinchus mystaceus

Platyrinchus coronatus

Platyrinchus platyrhynchos

Onychorhynchus coronatus

Terenotriccus erythrurus

Myiobius villosus

Myiobius barbatus

Myiotriccus ornatus

Myiophobus inornatus

Myiophobus ochraceiventris

Myiophobus fasciatus

Pyrrhomyias cinnamomea

Mitrephanes olivaceus

Contopus borealis (BM)

Contopus fumigatus

Contopus virens (BM)

Contopus sordidulus (BM)

Contopus cinereus

Empidonax alnorum (BM)

Lathrotriccus euleri

Cnemotriccus fuscatus

Undescribed tyrannid, cf.

Cnemotriccus ${ }^{*}$
$\mathrm{P}$

E Pyrocephalus rubinus

$X(1) \quad$ Ochthoeca cinnamomeiventris

$X(4) \quad$ Ochthoeca frontalis

E Ochthoeca pulchella

$X(3) \quad$ Ochthoeca rufipectoralis

X(3) Ochthoeca fumicolor

E Ochthoeca oenanthoides

E Ochthoeca leucophrys

E Ochthornis littoralis

E Myiotheretes striaticollis

X(1) Myiotheretes erythropygius

$X(1) \quad$ Myiotheretes rufipennis

E Myiotheretes fuscorufus

$X(1) \quad X o l m i s$ cinerea

E Xolmis velata

$\mathrm{X}(\mathrm{I}) \quad$ Xolmis irupero

$\mathrm{E} \quad$ Agriornis montana

X(1) Agriornis andicola

X(1) Agriornis microptera

$\mathrm{E} \quad$ Agriornis murina

X(3) Muscisaxicola maculirostris

X(1) Muscisaxicola fluviatilis

X(1) Muscisaxicola capistrata

X(1) Muscisaxicola rufivertex

E Muscisaxicola juninensis

X(1) Muscisaxicola albilora

E Muscisaxicola alpina

X(1) Muscisaxicola cinerea

X(1) Muscisaxicola albifrons

$\mathrm{E}$

$P$

E

E

$\mathrm{E}$

$\mathrm{X}(\mathrm{I}) \quad$ Knipolegus poecilurus

E Hymenops perspicillata

E Fluvicola pica

X(3) Arundinicola leucocephala

E Colonia colonus

E Alectrurus tricolor

E Gubernetes yetapa

$X(1) \quad$ Satrapa icterophrys

$X(3) \quad$ Hirundinea ferruginea

$X(1,3) \quad$ Machetornis rixosus

$X(1) \quad$ Attila cinnamomeus

Attila bolivianus

E

Attila spadiceus
E

$X(1)$

$\mathrm{E}$

E

E

$\mathrm{E}$

$\mathrm{E}$

E

$\mathrm{E}$

$X(1)$

E

$\mathrm{E}$

E

E

E

$\mathrm{E}$

$\mathrm{E}$

E

$\mathrm{E}$

E

E

E

$X(1)$

E

E

E

E

$X(2)$

$P$

E

E

$X(2)$

E

E

E

$P$

$X(1)$

$X(4)$

E

E

E

E

$X(1)$

E

E

$X(1)$

$X(1)$

$X(1)$ 
Casiornis rufa

Rhytipterna simplex

Laniocera hypopyrra

Sirystes sibilator

Myiarchus tuberculifer

Myiarchus swainsoni

Myiarchus ferox

Myiarchus cephalotes

Myiarchus tyrannulus

Pitangus lictor

Pitangus sulphuratus

Megarynchus pitangua

Myiozetetes cayanensis

Myiozetetes similis

Myiozetetes granadensis

Myiozetetes luteiventris

Conopias trivirgata

Myiodynastes chrysocephalus

Myiodynastes maculatus

Myiodynastes luteiventris (BM)

Legatus leucophaius

Empidonomus varius

Empidonomus

aurantioatrocristatus

Tyrannopsis sulphurea

Tyrannus albogularis

Tyrannus melancholicus

Tyrannus savana

Tyrannus tyrannus (BM)

Xenopsaris albinucha

Pachyramphus viridis

Pachyramphus versicolor

Pachyramphus castaneus

Pachyramphus polychopterus

Pachyramphus marginatus

Pachyramphus minor

Pachyramphus validus

Tityra cayana

Tityra semifasciata

Tityra inquisitor

\section{COTINGIDAE (20 species)}

Laniisoma (elegans) buckleyi

Phibalura flavirostris

Ampelion rubrocristatus

Ampelion rufaxilla

Pipreola intermedia

Pipreola arcuata
$\mathrm{X}(1,3) \quad$ Pipreola frontalis $\mathrm{E}$

X(1) Pipreola chlorolepidota E

$X(1) \quad$ Ampelioides tschudii E

$X(1) \quad$ Iodopleura isabellae $\quad X(1)$

$X(1) \quad$ Lipaugus vociferans $\quad X(1)$

$X(1) \quad$ Lipaugus uropygialis* ${ }^{*} \quad \mathrm{E}$

$X(1) \quad$ Porphyrolaema porphyrolaema $P$

$X(3) \quad$ Cotinga maynana $\quad X(1)$

$X(1) \quad$ Cotinga cayana E

$X(1) \quad$ Conioptilon mcilhenny $i^{*} \quad P$

$X(1) \quad$ Gymnoderus foetidus $\quad X(1)$

$X(1,3) \quad$ Querula purpurata $\quad X(1)$

$X(4) \quad$ Cephalopterus ornatus $\quad E$

$\mathrm{X}(\mathrm{1}, 3) \quad$ Rupicola peruviana $\mathrm{E}$

$X(1) \quad$ OXYRUNCIDAE (1 species)

$X(1)$ Oxyruncus cristatus E

E PIPRIDAE (17 species)

$\mathrm{X}(1,3) \quad$ Schiffornis major $\quad \mathrm{E}$

E Schiffornis turdinus $\quad X(1)$

E Piprites chloris $\quad X(1)$

$X(1) \quad$ Xenopipo atronitens $\mathrm{E}$

Heterocercus linteatus $\quad \mathrm{E}$

E Tyranneutes stolzmanni $\quad X(1)$

E Neopelma sulphureiventer E

E Machaeropterus pyrocephalus $\quad X(1)$

$X(1,3) \quad$ Chloropipo holochlora $\quad E$

E Chloropipo unicolor E

E Manacus manacus E

P Chiroxiphia pareola E

P Chiroxiphia boliviana E

E Pipra coronata X(1)

E Pipra fasciicauda $\quad X(1)$

$X(1,3) \quad$ Pipra rubrocapilla $\quad$ E

$X(1,3) \quad$ Pipra chloromeros $\quad X(1)$

$X(1)$

$X(1) \quad$ HIRUNDINIDAE (18 species)

$X(1) \quad$ Tachycineta albiventer $\quad X(1)$

$X(1) \quad$ Tachycineta leucorrhoa E

E Tachycineta leucopyga P

Progne tapera E

Progne subis (BM) P

Progne chalybea E

Progne modesta E

Notiochelidon murina E

Notiochelidon cyanolenca $\quad \mathrm{X}(1,3)$

Notiochelidon flavipes $\quad \mathrm{E}$

Atticora fasciata 
Neochelidon tibialis

Stelgidopteryx ruficollis

Alopochelidon fucata

Riparia riparia (BM)

Hirundo rustica (BM)

Hirundo andecola

Hirundo pyrrhonota (BM)

TROGLODYTIDAE (14 species)

Campylorhynchus turdinus

Odontorchilus branickit

Cinnycerthia peruana

Cistothorus platensis

Thryothorus genibarbis

Thryothorus leucotis

Troglodytes aedon

Troglodytes solstitialis

Henicorhina leucophrys

Microcerculus marginatus

Microcerculus bambla

Cyphorhinus aradus

Cyphorhinus thoracicus

Donacobius atricapillus

CINCLIDAE (1 species)

Cinclus leucocephalus

SYLVIIDAE (3 species)

Ramphocaenus melanurus

Microbates cinereiventris

Polioptila plumbea

\section{TURDIDAE (17 species)}

Myadestes ralloides

Entomodestes leucotis

Catharus fuscater

Catharus dryas

Catharus fuscescens (BM)

Catharus ustulatus (BM)

Platycichla leucops

Turdus chiguanco

Turdus fuscater

Turdus serranus

Turdus nigriceps

Turdus leucomelas

Turdus amaurochalinus

Turdus ignobilis

Turdus lawrencii

Turdus hauxzelli

Turdus albicollis

$P$

E

E

E

E

$\begin{array}{lll}\text { X(1) } & \text { MIMIDAE (2 species) } & \\ \mathrm{X}(1) & \text { Mimus saturninus } & \mathrm{E} \\ \mathrm{E} & \text { Mimus triurus } & \mathrm{E} \\ \mathrm{E} & \text { CORVIDAE (4 species) } & \\ \mathrm{E} & \text { Cyanolyca viridicyana } & \mathrm{E} \\ \mathrm{E} & \text { Cyanocorax cyanomelas } & \mathrm{X}(3) \\ \mathrm{E} & \text { Cyanocorax violaceus } & \mathrm{X}(1) \\ & \text { Cyanocorax yncas } & \mathrm{E}\end{array}$

$\mathrm{X}(1) \quad$ VIREONIDAE (8 species)

E Cyclarhis gujanensis

$\mathrm{E} \quad$ Vireolanius leucotis

$X(1,3)$

$\mathrm{E}$

$X(1,3)$

$\mathrm{P}$

E

$\mathrm{E}$

$X(1)$

$X(1)$

X(1) Hylophilus ochraceiceps

X(1) Anthus furcatus

$\mathrm{E} \quad$ Anthus lutescens

X(1) Anthus correndera

Anthus hellmayri

Anthus bogotensis

EMBERIZIDAE (49 species)

Zonotrichia capensis

P

Ammodramus humeralis

$X(2,3)$

$X(3)$

Ammodramus aurifrons

Phrygilus punensis

$X(1,3)$

E

$P$

Phrygilus fruticeti

Phrygilus unicolor

E

E

$P$

E

$\mathrm{E}$

$X(2)$

E

$X(2)$

$X(2)$

$\mathrm{P}$

E

E

E

$X(1)$

$X(3)$

E

$\mathrm{E}$ 
Sporophila americana

Sporophila lineola

Sporophila luctuosa

Sporophila caerulescens

Sporophila leucoptera

Sporophila minuta

Sporophila ruficollis

Sporophila castaneiventris

Sporophila hypochroma

Oryzoborus maximiliani

Oryzoborus angolensis

Catamenia analis

Catamenia inornata

Catamenia homochroa

Arremon taciturnus

Arremon flavirostris

Atlapetes rufinucha

Buarremon torquatus

Buarremon brunneinuchus

Coryphaspiza melanotis

Paroaria gularis

Pheucticus aureoventris

Caryothraustes humeralis

Saltator grossus

Saltator maximus

Saltator coerulescens

Cyanocompsa cyanoides

CATAMBLYRHYNCHIDAE (1 species)

Catamblyrhynchus diadema

THRAUPIDAE (91 species)

Schistochlamys melanopis

Conothraupis speculigera

Lamprospiza melanoleuca

Cissopis leveriana

Chlorornis riefferil

Chlorospingus ophthalmicus

Chlorospingus parvirostris

Chlorospingus canigularis

Hemispingus calophrys*

Hemispingus superciliaris

Hemispingus melanotis

Hemispingus xanthophthalmus

Hemispingus trifasciatus

Thlypopsis sordida

Thlypopsis ruficeps

Hemithraupis guira
P Hemithraupis flavicollis

$X(1)$

E Nemosia pileata

E Chlorothraupis carmioli

$X(1) \quad$ Eucometis penicillata

E Lanio versicolor

E Creurgops dentata

$X(3) \quad$ Tachyphonus cristatus

$X(1) \quad$ Tachyphonus rufiventer

E Tachyphonus luctuosus

E Trichothraupis melanops

X(1) Habia rubica

E Piranga flava

E Piranga rubra (BM)

E Piranga olivacea (BM)

$X(1) \quad$ Piranga leucoptera

$X(3) \quad$ Ramphocelus carbo

E Ramphocelus nigrogularis

E Thraupis episcopus

E Thraupis sayaca

E Thraupis palmarum

X(1) Thraupis cyanocephala

E Thraupis bonariensis

$X(\mathrm{~T}) \quad$ Buthraupis montana

$\mathrm{E} \quad$ Anisognathus igniventris

$X(1,3)$ Anisognathus flavinuchus

$X(1) \quad$ Iridosornis analis

$X(1,3) \quad$ Iridosornis jelskii

Delothraupis castaneoventris

Pipraeidea melanonota

E

Euphonia chlorotica

Euphonia laniirostris

Euphonia musica

$X(3) \quad$ Euphonia chrysopasta

$X(1) \quad$ Euphonia mesochrysa

$X(1) \quad$ Euphonia minuta

$X(1) \quad$ Euphonia xanthogaster

$\mathrm{E} \quad$ Euphonia rufiventris

E Chlorophonia cyanea

E Chlorochrysa calliparaea

E Tangara mexicana

E Tangara chilensis

E Tangara schrankii

E Tangara arthus

E Tangara xanthocephala

E Tangara chrysotis

E Tangara xanthogastra

E Tangara punctata

X(3) Tangara gyrola
$X(3)$

$\mathrm{E}$

E

$X(1,3)$

$\mathrm{E}$

$X(1)$

$\mathrm{E}$

$X(1)$

$\mathrm{E}$

$X(1)$

$\mathrm{E}$

$\mathrm{E}$

E

$\mathrm{E}$

$X(1,3)$

$X(1)$

$X(I)$

$X(3)$

$X(1,3)$

E

E

E

E

E

E

E

E

E

$X(3)$

$X(1,3)$

$X(1)$

$X(1)$

E

$X(1)$

$X(1)$

$X(1)$

$X(1)$

$\mathrm{E}$

$X(I)$

$X(1)$

$X(1)$

E

E

E

$X(1)$

E

E 


Tangara cayana
Tangara meyerdeschauenseei*
Tangara ruficervix
Tangara cyanotis
Tangara cyanicollis
Tangara nigrocincta
Tangara nigroviridis
Tangara vassorii
Tangara argyrofenges
Tangara viridicollis
Tangara velia
Tangara callophrys
Dacnis lineata
Dacnis flaviventer
Dacnis cayana
Chlorophanes spiza
Cyanerpes caeruleus
Cyanerpes cyaneus
Oreomanes fraseri
Diglossa baritula
Diglossa mystacalis
Diglossa carbonaria
Diglossa glauca
Diglossa caerulescens
Diglossa cyanea
Tersina viridis
Coereba flaveola

PARULIDAE (22 species)

Parula pitiayumi

Dendroica cerulea (BM)

Dendroica fusca (BM)

Geothlypis aequinoctialis

Oporornis agilis (BM)

Myioborus miniatus

Myioborus melanocephalus

Basileuterus bivittata

Basileuterus chrysogaster

Basileuterus flaveolus

Basileuterus luteoviridis

Basileuterus signatus

Basileuterus coronatus

Basileuterus culicivorus
E Basileuterus tristriatus E

E Phaeothlypis fulvicauda E

E Phaeothlypis rivularis $\quad X(\mathbf{1})$

E Conirostrum speciosum $X(3)$

E Conirostrum cinereum E

$\mathrm{X}(\mathrm{I})$ Conirostrum ferrugineiventre $\mathrm{E}$

E Conirostrum sitticolor E

E Conirostrum albifrons E

E ICTERIDAE (22 species)

$\mathrm{E} \quad$ Clypicterus oseryi $\mathrm{X}(1)$

$X(1) \quad$ Psarocolius decumanus $\quad X(1,3)$

$X(1) \quad$ Psarocolius atrovirens $\quad$ E

$X(1) \quad$ Psarocolius angustifrons $\quad X(1,3)$

$X(1) \quad$ Psarocolius bifasciatus $\quad X(1)$

$X(1) \quad$ Cacicus cela $X(1)$

$X(1) \quad$ Cacicus haemorrhous $\quad X(1)$

$X(1) \quad$ Cacicus leucoramphus $\quad E$

$\mathrm{E} \quad$ Cacicus solitarius $\mathrm{X}(1)$

$\mathrm{E} \quad$ Amblycercus holosericeus $\mathrm{E}$

$\mathrm{E} \quad$ Icterus cayanensis $\mathrm{X}(\mathrm{I})$

$\mathrm{E} \quad$ Icterus icterus $\mathrm{E}$

E Agelaius cyanopus E

E Agelaius xanthophthalmus E

$\mathrm{E} \quad$ Leistes militaris E

$\mathrm{X}(1,3) \quad$ Leistes superciliaris $\mathrm{X}(1)$

X(3) Amblyramphus holosericeus E

Gnorimopsar chopi E

Lampropsar tanagrinus $\quad \mathrm{E}$

Molothrus bonariensis $\quad \mathrm{X}(1)$

$\mathrm{X}(3) \quad$ Molothrus oryzivorus $\quad X(1)$

E Dolichonyx oryzivorus (BM) E

E CARDUELIDAE (6 species)

$\mathrm{X}(\mathbf{1}, 3)$ Carduelis crassirostris

$\mathrm{E} \quad$ Carduelis magellanica

$\mathrm{E} \quad$ Carduelis olivacea E

$\mathrm{E}(3) \quad$ Carduelis xanthogastra E

$\mathrm{X}(3) \quad$ Carduelis atrata $\quad \mathrm{X}(2)$

$\mathrm{E} \quad$ Carduelis uropygialis $\mathrm{E}$

E TOTAL SPECIES POSSIBLE 1,138

E Recorded so far (X) 515

E Expected (E) 573

E Possible (P) 50 